1 Department of Operative Dentistry, Endodontics and Dental Materials, Bauru School of Dentistry, University of Sao Paulo, Bauru, SP, Brazil

${ }^{2}$ Department of Comprehensive Care at Case Western Reserve University, School of Dental Medicine, Cleveland, $\mathrm{OH}$, USA
Corresponding author: Adilson Yoshio Furuse (iD) https://orcid.org/0000-0003-4705-6354 Email: furuse@usp.br Departamento de Dentística, Endodontia e Materiais Odontológicos Faculdade de Odontologia de Bauru Universidade de São Paulo Alameda Dr. Octávio Pinheiro Brisola, 9-75

CEP 17012-901

Telefone: (14) 3235-8000 Bauru - SP

Received: May 31, 2019

Accepted: January 22, 2020

\section{Color evaluation of white spot lesions treated with resin infiltration after water or grape juice storage}

\author{
Adilson Yoshio Furuse ${ }^{1, *}$, Constantino Fernandes \\ Neto ${ }^{1}$, Genine Moreira de Freitas Guimarães ${ }^{1}$, Bianca \\ Rodrigues Terrabuio ${ }^{1}$, Fabio Antonio Piola Rizzante², \\ Linda Wang
}

Aim: To evaluate the color stability of bovine enamel with artificial white spot lesions treated with resin infiltration (ICON) or remineralization with fluoride using two storage methods. Methods: Sixty incisors were submitted to artificial white spot lesion induced by demineralization-remineralization (DE-RE) cycling. Initial color was evaluated with CIE-Lab to measure $\Delta \mathrm{E}_{\mathrm{ab}}$. Demineralized teeth were divided according to the treatment of the white spot lesion ( $n=20): 1$ ) Remineralization with $2 \%$ neutral fluoride gel for 4min (control); 2) ICON application following manufacturer's recommendations; and 3) ICON with decreased drying time after the application of ethanol. After 24h, color was evaluated and samples were subdivided $(n=10)$ according to storage: 1) distilled water for 1 month; 2) grape juice for 10 min daily. After storage, color was evaluated. $L^{\star}, a^{*}$ and $b^{\star}$ data were analyzed by one-way ANOVA and $\triangle \mathrm{E}_{\mathrm{ab}}$ data by two-way ANOVA followed by Tukey's HSD $(a=0.05)$. Results: $L^{*}$ was affected by juice storage, and decreased when ICON was applied with decreased drying time after the ethanol application. The same behavior occurred with $a^{*}$ (increase with reduced drying time), while $b^{*}$ was not affected. For $\Delta \mathrm{E}_{\mathrm{ab}}$ significant differences were observed between groups ( $p=0.0219)$ and storage methods $(p=0.0007)$. There was no interaction effect $(p=0.1118)$. Remineralization with fluoride presented the lowest color changes after storage in water. Conclusion: Treatment of artificial carious lesions with resin infiltration presented greater color changes than fluoride remineralization after storage in both solutions in vitro.

Keywords: Dental caries. Dental enamel. Esthetics. 


\section{Introduction}

Dental caries is a common multifactorial disease that depends on host susceptibility, microorganism contamination, sugar rich diet and time for progression ${ }^{1}$. Enamel carious lesions are initially characterized by a pseudo intact surface layer and loss of minerals within the lesion body ${ }^{2}$. Its first clinical sign is the presence of opaque white spot lesions, which consist of non-cavitated subsurface lesions that can be treated conservatively using preventive approaches ${ }^{3}$. Among these approaches, the application of fluoride in the form of solution, gel or varnish are the most common in dental clinics, since it is cost accessible and easy to use ${ }^{4}$. In addition, the introduction of resinous materials to seal pits and grooves and to infiltrate white spot lesions brought the advantage of being an immediate treatment. However, studies with infiltrant in anterior teeth have questionable esthetic in masking white spot lesions in the long-term ${ }^{5-7}$

The appearance of a white spot occurs due to the imbalance in the processes of demineralization and remineralization that precedes the presence of early caries in ename $^{8}$. The increased porosity of the tissue allows air penetration within the enamel structure, resulting in altered optical properties of the dental surface that occur due to the difference in the refractive index between hydroxyapatite and air, which are 1.62 and 1.00 , respectively ${ }^{8}$. These changes cause the appearance of opaque white spots, which can be reversed through remineralization procedures. Because of this reversible characteristic, any white spot lesion should preferably be detected and treated, thus avoiding further loss of tooth structure and possible need of restoration? ${ }^{9}$.

The method for treatment of incipient caries with the use of infiltrating agents was introduced in the past decade. The product, known commercially as ICON (DMG, Hamburg, Germany), can be used on proximal or smooth areas and is a highly fluid resin, which improves its penetration and results in an increased microhardness of the lesion surface ${ }^{10}$. Besides, ICON promotes masking of white spot lesions based on changes in light scattering previously described ${ }^{11}$. ICON infiltrant is presented in a kit consisting of three separate steps: 1) ICON Etch (15\% hydrochloric acid), to remove the mineralized surface layer; 2) ICON Dry (99\% ethanol), to penetrate in the demineralized enamel and dentin, to remove water and to improve the penetration of resin monomers, and 3) ICON Infiltrant, a light-curing resin, composed of triethylene glycol dimethacrylate (TEGDMA). Through a non-invasive procedure, the resin penetrates the subsurface lesions without further damaging the tissues and prevents infiltration and diffusion of cariogenic acids inside the lesion, arresting caries progression ${ }^{12}$. The low viscosity of resin monomer and the high penetration coefficient are favorable characteristics of this material ${ }^{13}$. Clinical studies concluded that the use of infiltrating agents to control caries disease by non-operative approaches were more effective in reducing the progression of proximal caries than using invasive methods $s^{10,14}$.

Although resin infiltration of white spot lesions has been showing promising results ${ }^{10,15}$. The application of this material should be properly indicated, since the infiltrant acts differently in primary and permanent teeth due toto differences in the degree of miner- 
alization of each dentition ${ }^{16}$. Besides, that effect on white spots depends on the depth of penetration of the resin infiltrant ${ }^{17}$. Previous studies have reported limitations for the infiltrant to penetrate in lesions affecting dentin, attributing it to the presence of water in this tissue which restricts the penetration of resin monomers ${ }^{10}$. In addition, depending on the sorption and solubility of the resinous matrix, the long-term exposure to dyes and acidic solutions may degrade resin monomers through processes of swelling, plasticization, softening, oxidation, and hydrolysis, affecting its color stability $^{18}$. Based on these previous assumptions, the infiltrant application technique should be strictly performed according to the manufacturer's recommendation and further studies are necessary to determine the long-term benefits of the infiltrant application $^{19,20}$. Thereby, a hypothesis to be studied is whether possible variations and/or errors in the infiltrating technique could compromise the success of the final treatment. In addition, the esthetic aspects related to the use of ICON should also be further investigated, evaluating its effectiveness of resin infiltrants to mask white spots lesions in anterior teeth.

For this reason, it is important to consider the esthetic aspects after the application of infiltrants, since changes in color should be expected, considering the material aging, in which it is exposed to staining agents found in patient's diet. These extrinsic color changes can be found in foods and beverages, such as soft drinks, fruit juices and coffee. Due to the solubility and water absorption rate, the extrinsic coloring susceptibility of this resinous material may be linked to the resin matrix used ${ }^{13}$. Due to these factors, analyzing patient's habits, age and resin properties are of great relevance in order to avoid future esthetic problems. Furthermore, variations in the application technique related to inappropriate drying of the tooth may also play an important role in the final color. Thus, it is important to evaluate whether variations in the application technique and regarding exposure to staining solutions would influence the color stability of teeth with artificial white-spot lesions treated with ICON.

Therefore, the purpose of this in vitro study was to evaluate the color stability of bovine enamel with artificial white spot lesions, treated with infiltrating resin (ICON) or remineralization with neutral fluoride using different application techniques of ICON and two forms of storage (water or juice).

\section{Materials and Methods}

Sixty sound, free of cracks or malformations, bovine teeth had their color evaluated (initial sound) using a CIE-Lab based spectrophotometer (VITA Easyshade 3D Master, Vita Zahnfabrik, BädSackingen, Germany). The color readings were standardized by performing the analysis in the same room with controlled illumination by the same operator. The tip of the device was held perpendicular to the enamel surface and three readings were performed for each specimen. Subsequently, artificial white spot lesion was induced by demineralization-remineralization (DE-RE) cycling at $\mathrm{pH}$ 4.7 and $37^{\circ} \mathrm{C}$ for 7 days ${ }^{21}$. The bovine tooth samples were immersed in demineralizing solution during $6 \mathrm{~h}$ for 5 days and after immersed in remineralizing solution during $18 \mathrm{~h}$ in the last 2 days of cycling. The demineralizing and remineralizing solutions used in this process are composed respectively of: $2.0 \mathrm{mM} \mathrm{Ca}\left(\mathrm{NO}_{3}\right)_{2} \cdot 4 \mathrm{H}_{2} \mathrm{O}$, 2.0mM NaH${ }_{2} \mathrm{PO}_{4} \cdot 2 \mathrm{H}_{2} \mathrm{O}, 0.077 \mathrm{mM}$ acetate buffer, 0.02 ppm F (demineralizing solu- 
tion); and $1.5 \mathrm{mM} \mathrm{Ca}\left(\mathrm{NO}_{3}\right)_{2} \cdot 4 \mathrm{H}_{2} \mathrm{O}, 0.9 \mathrm{mM} \mathrm{NaH}_{2} \mathrm{PO}_{4} \cdot 2 \mathrm{H}_{2} \mathrm{O}, 150 \mathrm{mM} \mathrm{KCl}, 0.1 \mathrm{~mol} / \mathrm{L}$ tris buffer, $0.03 \mathrm{ppm} F$ (remineralizing solution). After the induction of artificial white spot lesion, a second color measurement was performed (initial white spot).

For the sake of confirming the white spot lesion formation, the color change caused DE-RE cycling $\left(\triangle \mathrm{E}_{\mathrm{ab}}\right)$ was calculated using the ClE-Lab color system from the individual values of $L^{*}, a^{*}$ and $b^{*}$, according to the following equation:

$$
\Delta \mathrm{E}_{\mathrm{ab}}=\left[\left(\Delta \mathrm{L}^{*}\right)^{2}+\left(\Delta \mathrm{a}^{*}\right)^{2}+\left(\Delta \mathrm{b}^{*}\right)^{2}\right]^{1 / 2}
$$

$\Delta L *, \Delta a *$ and $\Delta b$ * represent the differences between the readings of the color parameters before and after $\mathrm{pH}$ cycling. All teeth had $\Delta \mathrm{E}_{\mathrm{ab}}$ considered visible to the human eye being $\Delta \mathrm{E}_{\mathrm{ab}}>3$ (mean value of 4.85$)^{22}$.

Groups were divided according to the treatment of white spot lesions ( $n=20$ per group): 1) remineralization with neutral fluoride gel $2 \%$ (Flugel, DFL Indústria e Comércio SA, Rio de Janeiro, RJ, Brazil) for 4 min (control); 2) ICON (DMG do Brasil, São Paulo, SP, Brazil) application according to the manufacturer's recommendations; 3 ) ICON application with decreased drying time after the application of ethanol (ICON dry). For determination of the sample size, $a$ and $\beta$ errors of $5 \%$ and $20 \%$ were respectively set and a standard deviation of 1.5 obtained in the pilot study was used.

For group 1, the surface was cleaned with fine pumice and rubber cups, followed by application of thee neutral fluoride for 4 min. Excesses were removed with a microbrush. For groups 2 and 3 , the surface of the specimens was also cleaned, followed by acid etch with 15\% hydrochloric acid (ICON-Etch) for 2 min, washing with water and drying with air jets for 30s. Ethanol (ICON-Dry) was applied for 30s followed by drying with air for 30s (group 2) and for 5s (group 3). The infiltrator (ICON-Infiltrant) was applied to the surface twice, the first time for 3 min and the second time for 1 min according manufacturer's recommendations followed by photoactivation (VALO Cordless, Ultradent Products Inc., South Jordan, UT, USA) for 40s both application steps. The LED device was used in standard mode at $1000 \mathrm{~mW} / \mathrm{cm}^{2}$. A digital flat-response power-meter (New Port - model 2936-R; Irvine, CA, USA) was used to measure the power in $\mathrm{W}$ with the tip of the light source placed in close contact with the device. The irradiance (in $\mathrm{mW} / \mathrm{cm}^{2}$ ) was obtained by dividing the power (in $\mathrm{mW}$ ) by the area of the LED tip ${ }^{23}$. After photoactivation a third color reading was performed (ICONO).

The specimens were stored in artificial saliva ${ }^{24}$, composed of: $1.5 \mathrm{mM} / \mathrm{L} \mathrm{Ca}\left(\mathrm{NO}_{3}\right)_{2}$. $4 \mathrm{H}_{2} \mathrm{O}, 0.9 \mathrm{mM} / \mathrm{L} \mathrm{NaH}_{2} \mathrm{PO}_{4} .2 \mathrm{H}_{2} \mathrm{O}, 150 \mathrm{mM} / \mathrm{L} \mathrm{KCl}, 0.1 \mathrm{~mol} / \mathrm{L}$ tris buffering, $0.03 \mathrm{ppm} \mathrm{F}$ for $24 \mathrm{~h}$ to rehydrate the surface of the tooth and the infiltrant matrix and another color measurement was performed (ICON24h). Each group $(n=20)$ was subdivided into 2 subgroups ( $n=10$ per subgroup) according to the storage form: 1 ) distilled water for one month; 2) grape juice (Frupic, Ind. And Palazzos Ltda. Trade, Jaboticabal, SP, Brazil) for 10 minutes daily, followed by storage in distilled water for 23:50h for 5 days.

The color stability $(\triangle E)$ was calculated using the ClE-Lab color system to obtain $\triangle E_{a b}$ from the individual values of $L^{*}, a^{*}$ and $b^{*}$, according to the following equation: 
$\Delta \mathrm{E}_{\mathrm{ab}}=\left[\left(\Delta \mathrm{L}^{*}\right)^{2}+\left(\Delta \mathrm{a}^{*}\right)^{2}+\left(\Delta \mathrm{b}^{*}\right)^{2}\right]^{1 / 2}$

$\Delta L *, \Delta a *$ and $\Delta b *$ represent the differences between the readings of the color parameters. $\Delta \mathrm{E}_{\mathrm{ab}}$ represents the difference between the measurements obtained after artificial white spot lesion induction and in sound enamel. $\Delta \mathrm{E}_{\mathrm{J} / \mathrm{W}}$ stands for the difference between measurements taken after water or juice storage and values assessed after 24h on artificial saliva.

The data was evaluated for normality using Kolmogorov-Smirnov test. L*, a* and b* data were evaluated with One-way ANOVA and Tukey's HSD. $\triangle \mathrm{E}_{\mathrm{ab}}$ data were analyzed by Two-way ANOVA considering treatments considering treatments and storage methods as independent variables. Tukey's HSD was applied for multiple comparison. A global level of significance of $5 \%$ was adopted ( $a=0.05)$. Data was analyzed using the software Statistica 13.3 (Statsoft, Dell Software, Round Rock, TX, USA).

\section{Results}

$L^{*}, a^{*}$, and $b^{*}$ mean values and standard deviations are presented in Figures 1 to 3 , respectively. L* was mainly affected by the juice storage, and decreased when ICON was applied with decreased drying time after the application of ethanol. The same behavior occurred with a* (increase with reduced drying time), while b* was not affected. Analysis of variance of $\Delta \mathrm{E}_{\mathrm{ab}}$ is presented in Table 1. Means and standard deviations for $\Delta \mathrm{E}_{\mathrm{ab}}$ data are presented in Figure 4 and Table 2. Significant differences were observed between the groups ( $p=0.0217)$ and between storage methods ( $p=0.0007)$. For measurements made after storage in water/juice, $\Delta E_{a b}$ values were lower for samples treated with fluoride and ICON with recommended time, both stored in water. The highest $\triangle \mathrm{E}_{\mathrm{ab}}$ values was found for samples treated with ICON with recommended time stored in juice.

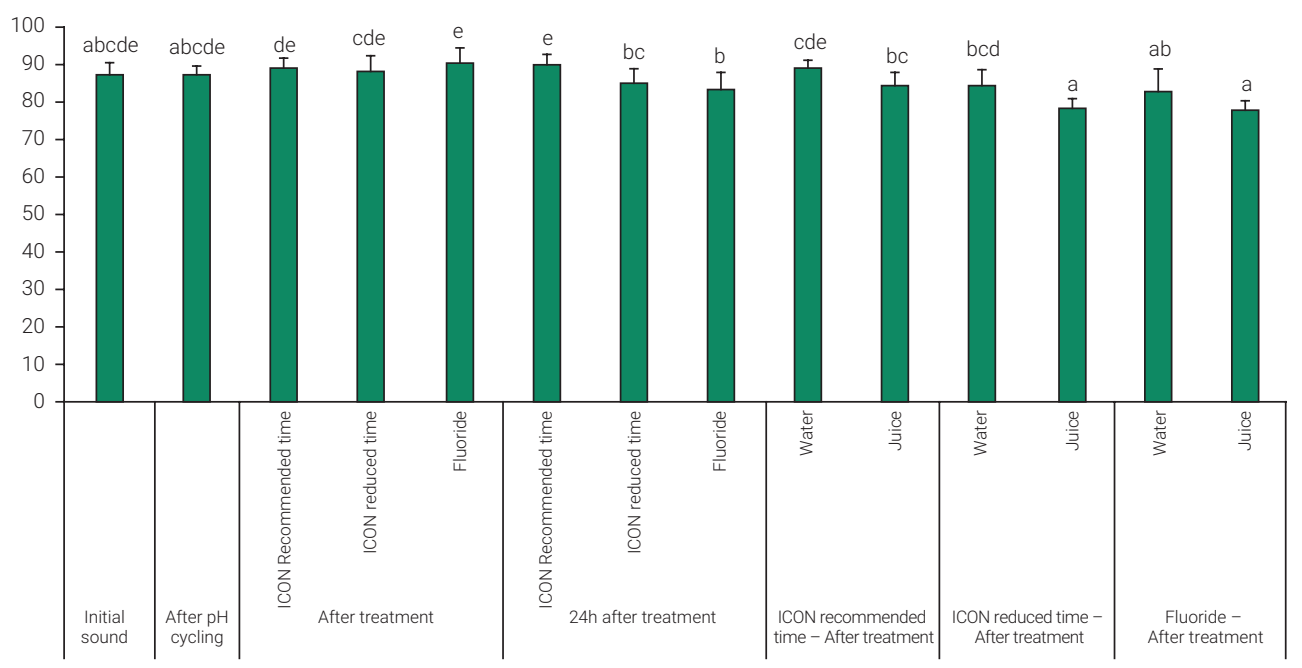

Figure 1. Graphic representation of $L^{*}$ mean values and standard deviations. Different letters indicate statistical significant difference $(p<0.05)$. 


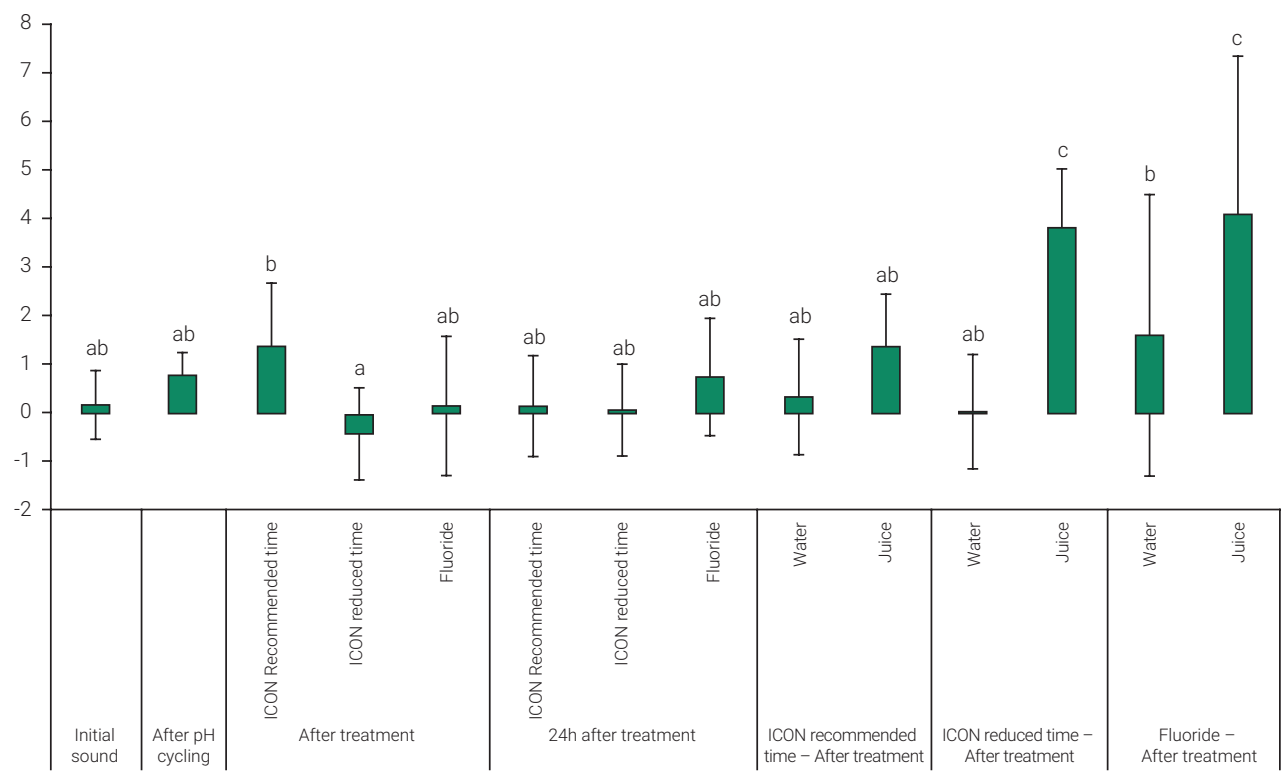

Figure 2. Graphic representation of $a^{*}$ mean values and standard deviations. Different letters indicate statistical significant difference $(p<0.05)$.

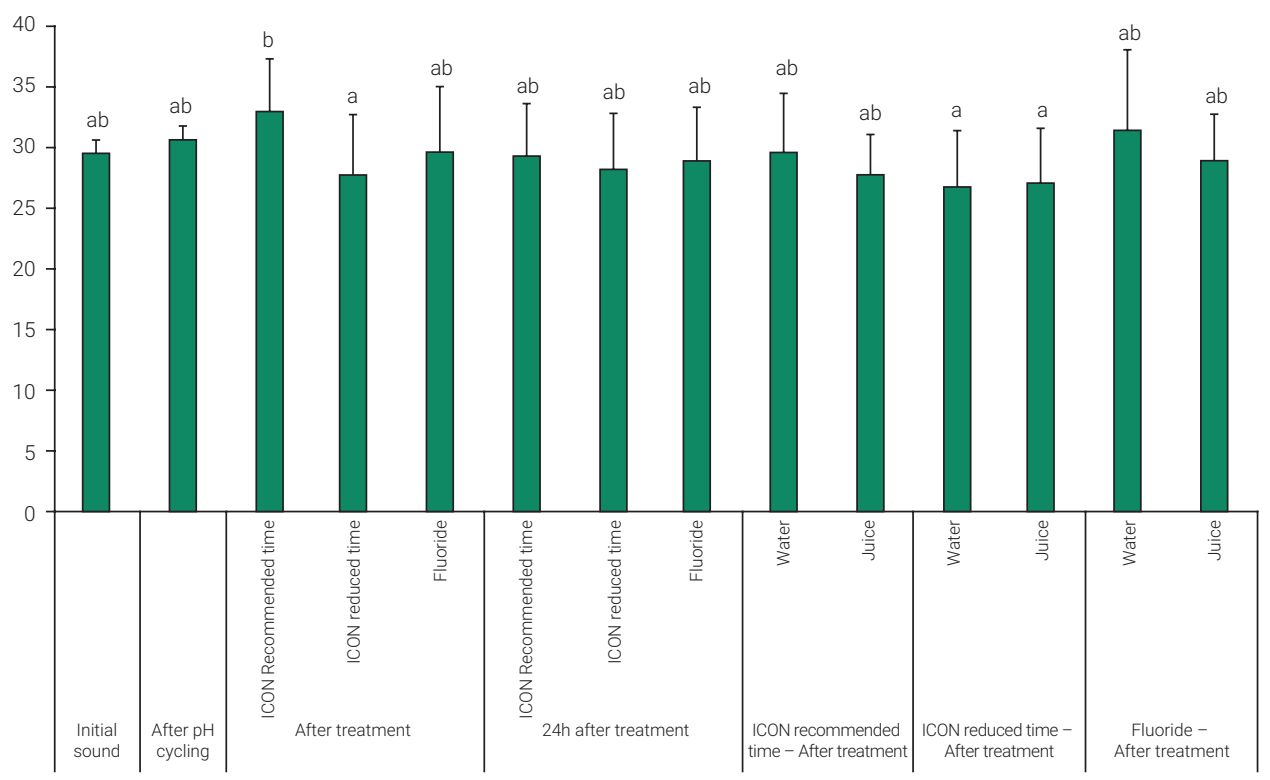

Figure 3. Graphic representation of $b^{\star}$ mean values and standard deviations. Different letters indicate statistical significant difference $(p<0.05)$.

All $\Delta \mathrm{E}_{\mathrm{ab}}$ means, which were taken after white spot lesion induction, presented values greater than 1 . There was no interaction effect between groups and storage methods $(p=0.1119)$. Treatments with fluoride have shown lower $\Delta E_{a b}$ values after storage methods when compared to both treatments with ICON. No statistically 
Table 1. Results from 2-way ANOVA of $\triangle \mathrm{E}_{\mathrm{ab}}$ means.

\begin{tabular}{lccccc}
\hline Variables of interest & $\begin{array}{c}\text { Degree of } \\
\text { Freedom }\end{array}$ & $\begin{array}{c}\text { Sum of } \\
\text { Squares }\end{array}$ & $\begin{array}{c}\text { Mean of } \\
\text { Squares }\end{array}$ & $\mathrm{F}$ & $\mathrm{p}$ \\
\hline Intercept & 1 & 2232.342 & 2232.342 & 197.6146 & 0 \\
\hline Group & 2 & 92.87969 & 46.43985 & 4.111016 & $0.021784^{*}$ \\
\hline Storage & 1 & 145.864 & 145.864 & 12.91239 & $0.000706^{*}$ \\
\hline Group*Storage & 2 & 49.89429 & 24.94714 & 2.208408 & 0.119704 \\
\hline Error & 54 & 610.0078 & 11.29644 & & \\
\hline
\end{tabular}

* Statistical significant difference for $\mathrm{p}<0.05$.

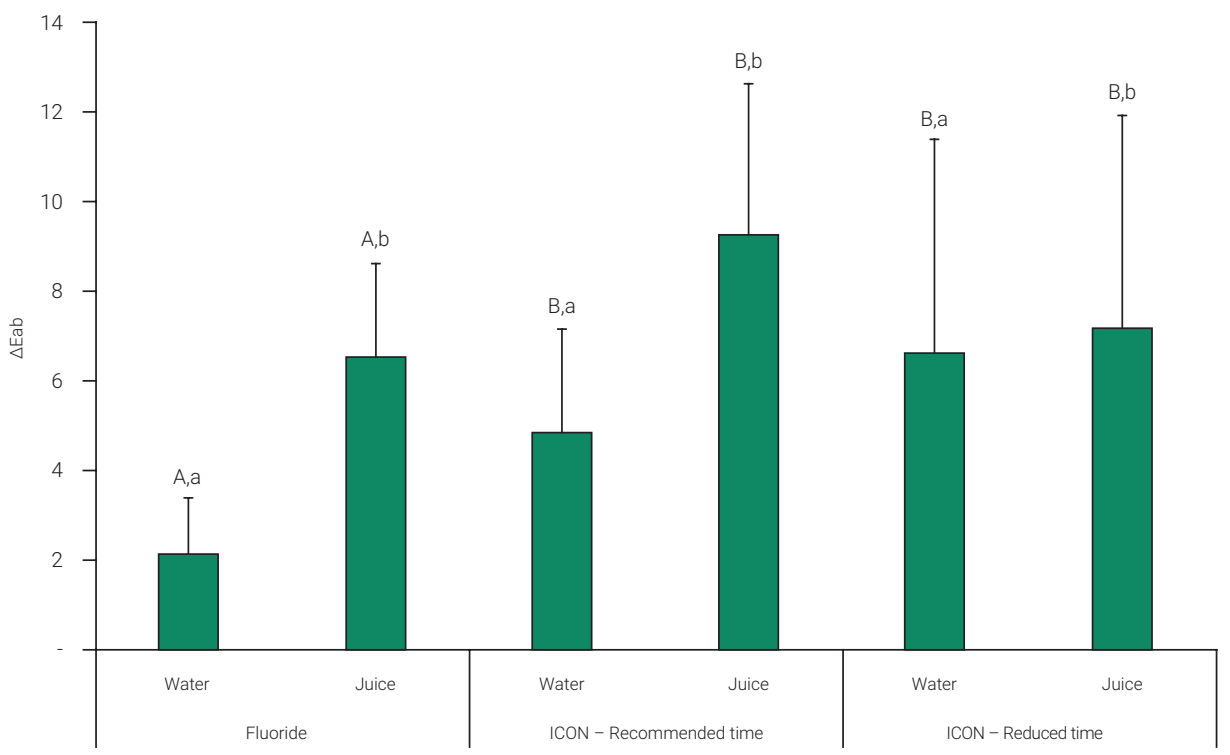

Figure 4. Graphic representation of $\Delta \mathrm{E}_{\mathrm{ab}}$. Uppercase letters indicate difference between groups of treatment (fluoride, ICON - Recommended time and ICON - Reduced time) and lowercase letters indicate difference between storage methods (water and juice).

Table 2. Mean $\Delta \mathrm{E}_{\mathrm{ab}}$ and standard deviation for treatment groups and storage methods.

\begin{tabular}{lcc}
\hline Group & Storage & $\Delta \mathrm{E}_{\mathrm{ab}}$ \\
\hline \multirow{2}{*}{ Fluoride } & Water & $2.15(1.24)^{\mathrm{A}, \mathrm{a}}$ \\
\hline \multirow{2}{*}{ Icon - Recommended time } & Juice & $6.53(2.09)^{\mathrm{A}, \mathrm{b}}$ \\
\hline \multirow{2}{*}{ Icon - Reduced time } & Water & $4.83(2.33)^{\mathrm{B}, \mathrm{a}}$ \\
\cline { 2 - 3 } & Juice & $9.25(3.38)^{\mathrm{B}, \mathrm{b}}$ \\
\cline { 2 - 3 } & Water & $6.64(4.73)^{\mathrm{B}, \mathrm{a}}$ \\
\hline
\end{tabular}

Uppercase letters indicate difference between groups of treatment and lowercase letters indicate difference between storage methods $(p<0.05)$.

significant difference was observed between ICON applications with reduced drying time. Storage in grape juice resulted in higher $\Delta \mathrm{E}_{\mathrm{ab}}$ mean values compared with storage in water. 


\section{Discussion}

Resin infiltration is currently indicated to mask defects in enamel staining, such as white spot lesions. In this case, ICON can prevent the progression of non-cavitated enamels caries $5,12,25$. This material improves the esthetic aspects of the lesion due to monomer infiltration through porosities of white spot lesions, decreasing their opacity $^{5}$. The present study was designed to evaluate the color stability of bovine teeth with artificial white spot lesions treated with remineralization (Flugel, DFL Indústria e Comércio SA) or resin infiltration (ICON, DMG) using two different application techniques.

The CIE-Lab represents a uniform color space with three distinct axes: $L^{*}$, $a^{*}$ and $b^{*}$. $L^{*}$ is the measure of brightness, and the closer to zero, the less brightness the object has and the closer to 100, the brighter. The negative value of $a^{*}$ indicates a measure of green color and, when positive, indicates a measure of red color. The positive $b^{*}$ is a measure of the yellow color and, when negative, of the color blue. When $a^{*}$ and $b^{*}$ are close to zero, the color is neutral ${ }^{6}$. The CIE-Lab was used in this study because it is able to evaluate the lightness-darkness, red/green and blue/yellow color measurements. Previous studies have demonstrated that such parameters are enough to evaluate white spot lesions, since these lesions are more opaque and whiter than healthy enamel. Although some studies use CIEDE2000 system $\left(\Delta E_{00}\right)$ to assess color measurements, the CIE-Lab system is still a reliable system used and has been used in recent studies to evaluate color stability of white spot lesions ${ }^{7,26}$. Based on this CIE-Lab system, it is possible to calculate the $\Delta \mathrm{E}_{\mathrm{ab}}$ and compare it with a visual perception already reported in the literature (color variation is imperceptible when $<1$, clinically acceptable when $\leq 3.3$ and unacceptable when above 3.3$)^{22}$. In the present study, color coordinates were obtained at several time intervals as described in the materials and methods section. However, since the aim was to address color changes through $\Delta \mathrm{L}^{*}, \Delta \mathrm{a}^{*}$, and $\Delta \mathrm{b}^{*}$ calculation after two protocols of ICON application as compared to fluoride remineralization, only $L^{*}$, $a^{*}$, and $b^{*}$ values collected $24 \mathrm{~h}$ after storage in artificial saliva were considered for $\Delta \mathrm{E}_{\mathrm{ab}}$ calculation. It should be noted that, immediately after the application of ICON and fluoride, samples were dehydrated, leading to altered measurements and for this reason these values were not applied to the $\Delta E_{a b}$ equation. Changes on color coordinates $L^{*}$, $a^{*}$, and $b^{*}$ were presented in figures 1 to 3 , which revealed significant differences in mean values for $L^{*}$ and $a^{*}$ when ICON was applied with decreased drying time after the application of ethanol. a values were also higher for samples treated with ICON reduced time after storage in juice. $L^{*}, a^{*}$, and $b^{*}$ values provide a clue to possible color changes, $\Delta \mathrm{E}_{\mathrm{ab}}$ values are more appropriate to indicate significant color changes visible to the human eye. Thereby, the present study was effective in inducing the formation of artificial white spot lesions, which can be verified by the value of $\Delta \mathrm{E}_{\mathrm{ab}}$ of 4.85 , which is greater than 3 for samples of all experimental groups and assess color differences between different treatments of white spot lesions.

The first hypothesis of this study was that a simple error in the application protocol of ICON, such as a marked decrease in the time of evaporation of ethanol after acid conditioning, could influence its color stability. This hypothesis, however, was not confirmed, since no statistically significant difference was observed between ICON 
experimental groups. As the application of ethanol has the purpose of dehydrating the surface and increase the penetration power of the infiltrate within the lesion due to the removal of water molecules from the enamel portions, an error in this step could decrease the subsequent penetration of the infiltrating agent or, eventually, disrupt its polymerization ${ }^{14}$. However, the decrease in ethanol evaporation time after the acid conditioning step did not influence the color stability of the infiltrator. This result can be possibly attributed to better penetration capabilities of TEGDMA-based resins $^{10}$, because it has a lower viscosity. A previous study accomplished by Gray and Shellis ${ }^{9}$ (2002) show that the efficacy of infiltration penetration depth is related to the increase of etching time. However, increasing the etching time can lead to wear of the enamel surface ${ }^{27}$. It should be noticed that not only the change in manufacturer's protocols could influence the curing efficiency of resin-based materials, but also variables related to the operator have a major impact in the final outcomes ${ }^{28}$.

Another hypothesis evaluated is that storage in grape juice would cause significant color change. The present study demonstrated that ICON is susceptible to staining when exposed to a natural agent such as grape juice. Despite adequate polymerization and polishing, resin-based materials are susceptible to pigmentation caused for common food substances and can produce significant discoloration of these materials ${ }^{13,29,30}$. This hypothesis was confirmed, since all $\Delta E_{W / J}$ values in which ICON was applied were higher than 3.3 units, agreeing with the work of Borges et al. ${ }^{13}$ (2014) which explains the clinically unacceptable color change after exposure to staining solutions. In general, all groups and subgroups had $\Delta \mathrm{E}_{\mathrm{ab}}$ greater than 3.3 units, except when fluoride was applied followed by storage in water. This means that storage in water caused a color change in resin-based materials that can be considered clinically significant and noticeable, whilst storage in grape juice induced color alterations for all experimental groups. These results are in agreement with other studies which demonstrated significant changes in color after coloring processes related to storage in pigmented solutions $6,26,29,31$.

These changes can be explained by the composition of the ICON, since it has TEGDMA on this matrix. TEGDMA monomer has high sorption of water and has a hydrophilic behavior in comparison with other monomers, which can either facilitate its depth of penetration in the white spot lesion and increase its susceptibility to degradation in water ${ }^{14,32,33}$. As a result it can be assumed that the infiltrant can easily absorb the dyes present in beverages and food, affecting its color stability ${ }^{34}$. Additionally, the sole absorption of water in the resinous materials matrix could be enough to cause color changes, which are illustrated in figure 1. Trying to avoid this effect, some authors suggested that the polishing of the specimens could minimize the staining effect ${ }^{13,14}$. However, the polishing alternative may result in unnecessary enamel wear caused by abrasion ${ }^{34}$. An alternative would be to perform a bleaching procedure, which does not promote the removal of enamel ${ }^{34}$.

Resin infiltration was shown to reliably mask artificial caries lesions in vitro, following the trend of a previous studies ${ }^{7,35}$. Within the limitation of this in vitro experiment, the results obtained from the present study could be of clinical relevance. A decrease in the ethanol evaporation time after an acid conditioning stage did not influence the color stability of the infiltrator. However, $L^{*}$ and $a^{*}$ were affected, without influenc- 
ing $\triangle \mathrm{E}$. Additionally, this study did not evaluate the infiltrator penetration depth. Further studies could be conducted evaluating the color changes occurring in the resin matrix maturation of ICON itself, without the influence of environmental factors, such as saliva, food dyes, water, dental structure or ions from oral cavity and the effect of infiltrator penetration depth on color stability.

Both materials tested showed significant color changes after exposure to staining dyes. It is assumed that while the ICON may help the initial aesthetic problem associated with white spot lesions, the resin may suffer from spotting over the time, especially when the patient ingests foods and beverages containing dyes. For this reason, the use of fluoride remineralization seems to be a good alternative, since it is clinically easy to perform and provides better long-term color stability in comparison to resin infiltration. ICON on the other hand, is known to provide satisfactory esthetic masking of the white spot lesion in an immediate one session application, requiring further polishing in future sessions to maintain its effectiveness. Therefore, such factors should be considered by the clinicians when choosing the treatment of white spot carious lesions.

Within the limitations of an in vitro study, the use of fluoride is preferable for treating white spot lesions rather than the application of resinous infiltrant because it's ability to prevent staining. Furthermore, the decrease in the ethanol evaporation time did not influence the color stability of the infiltrator, allowing the reduction of this clinical step time. More in vivo studies are necessary to validate the potential of ICON resin infiltration technique in providing color stability for white spot lesion treatment, since other clinical factors may be related.

\section{Acknowledgements}

This study was financed in part by the Coordenação de Aperfeiçoamento de Pessoal de Nível Superior - Brasil (CAPES) - Finance Code 001 and Conselho Nacional de Desenvolvimento Científico e Tecnológico/ PIBIC: 145207/2014-5.

\section{References}

1. Keyes PH. The infectious and transmissible nature of experimental dental caries. Findings and implications. Arch Oral Biol. 1960 Mar;1:304-20.

2. Askar H, Lausch J, Dörfer CE, Meyer-Lueckel H, Paris S. Penetration of micro-filled infiltrant resins into artificial caries lesions. J Dent. 2015 Jul;43(7):832-8. doi: 10.1016/j.jdent.2015.03.002.

3. Buzalaf MAR, Pessan JP, Honorio HM, Ten Cate JM. Mechanisms of action of fluoride for caries control. Monogr Oral Sci. 2011;22:97-114. doi: 10.1159/000325151.

4. Palacio R, Shen J, Vale L, Vernazza CR. Assessing the cost-effectiveness of a fluoride varnish programme in Chile: the use of a decision analytic model in dentistry. Community Dent Oral Epidemiol. 2019 Jun;47(3):217-24. doi: 10.1111/cdoe.12447.

5. Abbas BA, Marzouk ES, Zaher AR. Treatment of various degrees of white spot lesions using resin infiltration-in vitro study. Prog Orthod. 2018 Aug 6;19(1):27. doi: 10.1186/s40510-018-0223-3.

6. Cohen-Carneiro F, Pascareli AM, Christino MR, Vale HF, Pontes DG. Color stability of carious incipient lesions located in enamel and treated with resin infiltration or remineralization. Int J Paediatr Dent. 2014 Jul;24(4):277-85. doi: 10.1111/ipd. 12071. 
7. Knösel M, Eckstein A, Helms H-J. Long-term follow-up of camouflage effects following resin infiltration of post orthodontic white-spot lesions in vivo. Angle Orthod. 2019 Jan;89(1):33-39. doi: 10.2319/052118-383.1.

8. Kidd E, Fejerskov O. What constitutes dental caries? Histopathology of carious enamel and dentin related to the action of cariogenic biofilms. J J Dent Res. 2004;83 Spec No C:C35-8.

9. Gray G, Shellis P. Infiltration of resin into white spot caries-like lesions of enamel: an in vitro study. Eur J Prosthodont Restor Dent. 2002 Mar; 10(1):27-32.

10. Paris S, Schwendicke F, Seddig S, Müller W-D, Dörfer C, Meyer-Lueckel H. Micro-hardness and mineral loss of enamel lesions after infiltration with various resins: influence of infiltrant composition and application frequency in vitro. J Dent. 2013 Jun;41(6):543-8. doi: 10.1016/j.jdent.2013.03.006.

11. Manoharan V, Arun Kumar S, Arumugam SB, Anand V, Krishnamoorthy S, Methippara JJ. Is Resin Infiltration a Microinvasive Approach to White Lesions of Calcified Tooth Structures?: a systemic review. Int J Clin Pediatr Dent. 2019 Jan-Feb;12(1):53-58. doi: 10.5005/jp-journals-10005-1579.

12. Paris S, Hopfenmuller W, Meyer-Lueckel H. Resin infiltration of caries lesions: an efficacy randomized trial. J Dent Res. 2010 Aug;89(8):823-6. doi: 10.1177/0022034510369289.

13. Borges A, Caneppele T, Luz M, Pucci C, Torres C. Color stability of resin used for caries infiltration after exposure to different staining solutions. Oper Dent. 2014 Jul-Aug;39(4):433-40. doi: 10.2341/13-150-L.

14. Paris S, Schwendicke F, Keltsch J, Dorfer C, Meyer-Lueckel H. Masking of white spot lesions by resin infiltration in vitro. J Dent. 2013 Nov;41 Suppl 5:e28-34. doi: 10.1016/j.jdent.2013.04.003.

15. Belli R, Rahiotis C, Schubert EW, Baratieri LN, Petschelt A, Lohbauer U. Wear and morphology of infiltrated white spot lesions. J Dent. 2011 May;39(5):376-85. doi: 10.1016/j.jdent.2011.02.009.

16. Wilson P, Beynon A. Mineralization differences between human deciduous and permanent enamel measured by quantitative microradiography. Arch Oral Biol. 1989;34(2):85-8.

17. Yoo HK, Kim SH, Kim SI, Shin YS, Shin SJ, Park JW. Seven-year follow-up of resin infiltration treatment on noncavitated proximal caries. Oper Dent. 2019 Jan/Feb;44(1):8-12. doi: 10.2341/17-323-L.

18. Silva TMD, Sales A, Pucci CR, Borges AB, Torres CRG. The combined effect of food-simulating solutions, brushing and staining on color stability of composite resins. Acta Biomater Odontol Scand. 2017 Jan 16;3(1):1-7. doi: 10.1080/23337931.2016.1276838.

19. Domejean S, Ducamp R, Leger S, Holmgren C. Resin infiltration of non-cavitated caries lesions: a systematic review. Med Princ Pract. 2015;24(3):216-21. doi: 10.1159/000371709.

20. Swamy DF, Barretto ES, Mallikarjun SB, Dessai SSR. In vitro evaluation of resin infiltrant penetration into white spot lesions of deciduous molars. J Clin Diagn Res. 2017 Sep;11(9):ZC71-4. doi: 10.7860/JCDR/2017/28146.10599.

21. Vieira AE, Delbem ACB, Sassaki KT, Rodrigues E, Cury JA, Cunha RF. Fluoride dose response in pH-cycling models using bovine enamel. Caries Res. 2005 Nov-Dec;39(6):514-20.

22. Seghi RR, Johnston WM, O'Brien WJ. Performance assessment of colorimetric devices on dental porcelains. J Dent Res. 1989 Dec;68(12):1755-9.

23. Andreatta LM, Furuse AY, Prakki A, Bombonatti JF, Mondelli RF. Pulp chamber heating: an in vitro study evaluating different light sources and resin composite layers. Braz Dent $\mathrm{J}$. 2016 Oct-Dec;27(6):675-80. doi: 10.1590/0103-6440201600328.

24. Francisconi L, Honório HM, Rios D, Magalhães A, Machado MdA, Buzalaf MAR. Effect of erosive pH cycling on different restorative materials and on enamel restored with these materials. Oper Dent. 2008 Mar-Apr;33(2):203-8. doi: 10.2341/07-77. 
25. Horuztepe SA, Baseren M. Effect of resin infiltration on the color and microhardness of bleached white-spot lesions in bovine enamel (an in vitro study). J Esthet Restor Dent. 2017 Sep;29(5):378-85. doi: 10.1111/jerd.12308.

26. Ceci M, Rattalino D, Viola M, Beltrami R, Chiesa M, Colombo M, et al. Resin infiltrant for non-cavitated caries lesions: evaluation of color stability. J Clin Exp Dent. 2017 Feb 1;9(2):e231-e237. doi: $10.4317 /$ jced.53110.

27. Arnold WH, Haddad B, Schaper K, Hagemann K, Lippold C, Danesh G. Enamel surface alterations after repeated conditioning with $\mathrm{HCl}$. Head Face Med. 2015 Sep 25;11:32. doi: 10.1186/s13005-015-0089-2.

28. Soares CJ, Bragança GFd, Pereira RAdS, Rodrigues MdP, Braga SSL, Oliveira LRS, et al. Irradiance and radiant exposures delivered by LED light-curing units used by a left and right-handed operator. Braz Dent J. 2018 May-Jun;29(3):282-9. doi: 10.1590/0103-6440201802127.

29. Rey N, Benbachir N, Bortolotto T, Krejci I. Evaluation of the staining potential of a caries infiltrant in comparison to other products. D Dent Mater J. 2014;33(1):86-91.

30. Silva LO, Signori C, Peixoto AC, Cenci MS, Faria ESAL. Color restoration and stability in two treatments for white spot lesions. Int J Esthet Dent. 2018;13(3):394-403.

31. Villalta P, Lu H, Okte Z, Garcia-Godoy F, Powers JM. Effects of staining and bleaching on color change of dental composite resins. J Prosthet Dent. 2006 Feb;95(2):137-42.

32. Fonseca AS, Labruna Moreira AD, de Albuquerque PP, de Menezes LR, Pfeifer CS, Schneider LF. Effect of monomer type on the CC degree of conversion, water sorption and solubility, and color stability of model dental composites. Dent Mater. 2017 Apr;33(4):394-401. doi: 10.1016/j.dental.2017.01.010.

33. Sideridou ID, Karabela MM, Bikiaris DN. Aging studies of light cured dimethacrylate-based dental resins and a resin composite in water or ethanol/water. Dent Mater. 2007 Sep;23(9):1142-9.

34. Araújo G, Naufel F, Alonso R, Lima D, Puppin-Rontani R. Influence of staining solution and bleaching on color stability of resin used for caries infiltration. Oper Dent. 2015 Nov-Dec;40(6):E250-6. doi: 10.2341/14-290-L.

35. Theodory TG, Kolker JL, Vargas MA, Maia RR, Dawson DV. Masking and penetration ability of various sealants and ICON in artificial initial caries lesions in vitro. J Adhes Dent. 2019;21(3):265-72 doi: 10.3290/j.jad.a42520. 